\title{
Magnetotransport properties of InMnSb magnetic semiconductor thin films
}

\author{
J. A. Peters, N. D. Parashar, N. Rangaraju, and B. W. Wessels* \\ Materials Research Center, Department of Materials Science and Engineering, Northwestern University, Evanston, Illinois 60208, USA
}

(Received 8 December 2009; revised manuscript received 8 October 2010; published 16 November 2010)

\begin{abstract}
We report on the magnetotransport properties of epitaxial thin films of $\operatorname{In}_{1-x} \mathrm{Mn}_{x} \mathrm{Sb}$ dilute magnetic semiconductor grown by metal-organic vapor-phase epitaxy. At temperatures below $10 \mathrm{~K}$, a negative magnetoresistance dominates the magnetotransport that is attributed to spin-dependent scattering by localized magnetic moments. Above $10 \mathrm{~K}$, the magnetoresistance is positive and is well described by a two-band model consisting of spin-split hybridized $p-d$ subbands with different conductivities. Hall effect measurements show an anomalous behavior that persists up to room temperature, providing an indication of ferromagnetic order. In addition, magnetization measurements reveal distinct hysteresis loops at room temperature which confirms the ferromagnetism of the films.
\end{abstract}

DOI: 10.1103/PhysRevB.82.205207

PACS number(s): 71.55.Eq, 75.47.-m, 75.50.Pp, 75.70.Ak

\section{INTRODUCTION}

III-Mn-V dilute magnetic semiconductors (DMS) are of interest because of their potential application in the emerging field of spintronics, where the control of the spin state of carriers provides an important mechanism for future electronic devices. ${ }^{1}$ These magnetic materials possess unique characteristics that include the potential for externally controlled ferromagnetism, a high degree of spin polarization of carriers, and a wide variety of spin-dependent transport properties. ${ }^{2-4}$ The strong spin-dependent coupling between the bands and localized states account for the outstanding properties of DMS. ${ }^{5}$ This coupling gives rise to giant spin splitting of the electronic states and spin-disorder scattering.

The presence of local magnetic moments and, in particular, the exchange interaction between carriers and local moments has been known to dramatically modify carrier transport. The strong coupling between band and localized states leads to large magnetoresistive effects including both negative and positive magnetoresistance (MR). ${ }^{5}$ Several theoretical models have been proposed that describe the origin of the magnetoresistance. A theory of spin-disorder scattering by thermodynamic fluctuations of magnetic atoms was developed for ordered magnetic semiconductors ${ }^{6}$ based on an earlier theory of scattering in magnetic metal alloys. ${ }^{7}$ The presence of an external magnetic field suppresses the fluctuations of the local magnetization (due to magnetic moments of the magnetic ions) and leads to decreased scattering and a negative magnetoresistance. Positive magnetoresistance on the other hand has been attributed to increased scattering of carriers by localized magnetic moments due to an increase in the magnitude of the local potential due to the Zeeman effect $^{8-10}$ The increase in spin-dependent scattering by the external magnetic field is especially large for semiconductors with electrically active magnetic impurities. ${ }^{9}$

Magnetotransport of In-Mn-V DMS has been previously investigated where $\mathrm{Mn}$ is both the source of localized magnetic moments and free carriers. InMnAs grown by lowtemperature molecular-beam epitaxy (LT-MBE) shows a negative magnetoresistance at $3.5 \mathrm{~K}$ for $\mathrm{In}_{0.987} \mathrm{Mn}_{0.013} \mathrm{As}$ (Ref. 11) and $4.2 \mathrm{~K}$ for InMnAs/GaSb heterojunctions. ${ }^{12} \mathrm{In}$ the case of the heterojunctions, where $T_{\mathrm{C}}=35 \mathrm{~K}$, negative magnetoresistance is not observed above a $T_{\mathrm{C}}$ of $42 \mathrm{~K}$. The presence of magnetic polarons qualitatively explains the negative magnetoresistance observed at low temperatures in InMnAs grown by LT-MBE. ${ }^{13,14}$ The applied magnetic field aligns the magnetic moments of the substitutional Mn impurities, thereby making the polarons more mobile and increasing the conductivity.

Low-field magnetoresistance of InMnAs thin films grown by metal-organic vapor-phase epitaxy (MOVPE) has been previously measured up to $14 \mathrm{~K}$, revealing a negative magnetoresistance that was attributed to spin-selective scattering of carriers in an impurity band. ${ }^{15}$ At temperatures above 14 $\mathrm{K}$, however, a small positive magnetoresistance was observed. In a subsequent study, a positive magnetoresistance at higher temperatures was observed in thin films of InMnAs grown by MOVPE. ${ }^{16}$ The positive magnetoresistance at low fields $(<1 \mathrm{~T})$ and at temperatures above $17 \mathrm{~K}$ was attributed to conduction via spin-split hybridized $p$ - $d$ subbands with different conductivities and mobilities. The splitting of the band and the changes in the density of states result in different carrier populations in the two spin subbands. These subbands have different conductivities and mobilities for majority and minority spin carriers. Increased scattering of spinpolarized carriers due to changes in the local Zeeman potential leads to the positive magnetoresistance.

Magnetotransport studies on InMnSb thin films grown by LT-MBE have been previously reported. ${ }^{17,18}$ Initial measurements on $\operatorname{In}_{1-x} \mathrm{Mn}_{x} \mathrm{Sb}$ films indicate that scattering of carriers by isolated $\mathrm{Mn}^{2+}$ ions is the dominant mechanism, leading to a negative magnetoresistance for temperatures up to $12 \mathrm{~K} .{ }^{19}$ These LT-MBE alloys show well-defined magnetic hysteresis loops and an anomalous Hall effect (AHE). The Curie temperature $\left(T_{\mathrm{C}}\right)$ measured in these samples is very low, on the order of 7-20 K. Recently, however, we have shown that single phase $\mathrm{In}_{1-x} \mathrm{Mn}_{x} \mathrm{Sb}$ films grown by MOVPE are ferromagnetic at room temperature with a $T_{\mathrm{C}}$ in excess of 400 $\mathrm{K} .{ }^{20}$ Ferromagnetism was supported by field-cooled (FC) and zero-field-cooled (ZFC) magnetization measurements and by clear hysteresis in the anomalous Hall resistivity. The high $T_{\mathrm{C}}$ is attributed to carrier-mediated ferromagnetism involving $\mathrm{Mn}$ and its complexes that form shallow or resonant electronic states through correlated substitution in the semiconductor host. 
In the present work, we report on magnetoresistive properties of single-phase, semiconducting $\operatorname{In}_{1-x} \mathrm{Mn}_{x} \mathrm{Sb}$ thin films grown by MOVPE. We focus on the spin-dependent scattering processes responsible for the magnetoresistance observed in the films. We find that at temperatures below $10 \mathrm{~K}$ a negative magnetoresistance dominates the magnetotransport while above $10 \mathrm{~K}$ the magnetoresistance is positive. The negative magnetoresistance observed at low temperatures is attributed to scattering of spin-polarized holes by magnetic $\mathrm{Mn}^{2+}$ ions. For the observed positive magnetoresistance, a two-band model is proposed where the bands result from the strong $p$ - $d$ exchange interaction. The $p$ - $d$ exchange results in a splitting of the valence band into subbands for the two spin directions. Analytical expressions were developed that describe the behavior of magnetoresistance with field and temperature. From the MR measurements the carrier distribution and spin polarization were calculated. In addition, we present results of the Hall effect measurements that exhibit distinct hysteresis loops and an anomalous Hall resistivity even at room temperature. The transport properties are consistent with the presence of ferromagnetic order in these phase pure films.

\section{EXPERIMENT}

$\mathrm{In}_{1-x} \mathrm{Mn}_{x} \mathrm{Sb}$ thin films were grown using atmospheric pressure MOVPE on semi-insulating GaAs (100) substrates. The films were deposited at a substrate temperature of $400{ }^{\circ} \mathrm{C}$ at a typical growth rate of $\sim 330 \mathrm{~nm} / \mathrm{h}$. The thickness of the films was on the order of $300-550 \mathrm{~nm}$. Details of the growth procedure are reported elsewhere. ${ }^{20}$ The $\mathrm{In}_{1-x} \mathrm{Mn}_{x} \mathrm{Sb}$ films studied have a $\mathrm{Mn}$ concentration of $x$ $=0.02$ and 0.035 (herein referred to as $2 \%$ and $3.5 \% \mathrm{Mn}$ samples). The manganese concentration was determined by energy-dispersive x-ray spectroscopy using a beam energy of $25 \mathrm{keV}$ and a probe current of $30 \mathrm{~mA}$. The phase purity and the lattice constant of the films were determined by double crystal x-ray diffraction using $\mathrm{Cu} K \alpha_{1}$ radiation. X-ray diffraction (XRD) and high-resolution transmission electron microscopy (TEM) indicate that the films are single phase with no secondary phases present. ${ }^{21}$ In addition, extended $\mathrm{X}$-ray absorption fine-structure (EXAFS) analysis indicates that Mn substitutes for In and has tetrahedral coordination as would be expected for a single-phase film with a zinc-blende structure. $^{20-22}$

Magnetic properties of the films were measured with a superconducting quantum interference device (SQUID) magnetometer. For analysis, the diamagnetic contribution from the substrate and holder was subtracted from the total sample magnetization. For magnetotransport studies, Hall bars were patterned on the samples using standard photolithography and wet chemical etching. A gold wire was attached to each terminal by soldering indium dots on the pads of the Hall bar to make electrical contact. Magnetotransport properties were measured at the National High Magnetic Field Laboratory where a superconducting magnet with a variable temperature insert was used to sweep the magnetic field up to $18 \mathrm{~T}$ for temperatures ranging from 1.4 to $298 \mathrm{~K}$. Longitudinal $\left(R_{\mathrm{xx}}\right)$ and Hall $\left(R_{\mathrm{xy}}\right)$ resistances were measured using the standard four-probe lock-in technique with a $100 \mu \mathrm{A}$ drive current. The magnetic field was applied perpendicular to the plane of the sample. The $\operatorname{In}_{1-x} \mathrm{Mn}_{x} \mathrm{Sb}$ films were determined to be $p$ type from the Hall data.

\section{THEORY}

The observed negative magnetoresistance in dilute magnetic alloys at low temperature was initially explained in terms of the scattering of carriers by localized moments. ${ }^{23}$ Upon application of an external magnetic field, the localized magnetic moments in the alloy become ordered. Consequently, carrier scattering is reduced resulting in a decrease in resistivity that is proportional to the square of the magnetization $M$. However, while the qualitative features of the negative magnetoresistance at low fields were consistent with the localized magnetic moment model, quantitative disagreements existed. This is because the localized moment theory, and that of a similar model proposed later, ${ }^{24}$ was based on calculations involving only the second-order perturbation expansion of the exchange Hamiltonian. Hence, a modified theory was proposed that takes into account higherorder terms of the perturbation expansion. ${ }^{25}$ The negative magnetoresistance is then given by ${ }^{25}$

$$
\frac{\Delta \rho}{\rho}=-a^{2} \ln \left(1+b^{2} H^{2}\right),
$$

where $a$ and $b$ include the physical characteristics of the exchange interaction and $H$ is the applied magnetic field.

In the present work, we describe the observed negative magnetoresistance using the semiempirical expression given in Eq. (1). This equation is based on the third-order expansion of the exchange Hamiltonian and on the logarithmic temperature dependence of the zero-field resistivity of dilute magnetic alloys. ${ }^{23,26}$ In particular, the coefficients $a$ and $b$ in Eq. (1) are given by

$$
a=A_{1} J D\left(\varepsilon_{\mathrm{F}}\right)\left[S(S+1)+\left\langle M^{2}\right\rangle\right]
$$

and

$$
b^{2}=\left[1+4 S^{2} \pi^{2}\left(\frac{2 J D\left(\varepsilon_{\mathrm{F}}\right)}{g}\right)^{4}\right]\left(\frac{g \mu_{B}}{\alpha k_{B} T}\right)^{2} .
$$

Here, $J$ is the exchange interaction energy, $D\left(\varepsilon_{\mathrm{F}}\right)$ is the density of states at the Fermi level, $\left\langle M^{2}\right\rangle$ is the average magnetization squared, $\mu_{B}$ is the Bohr magneton, and $\alpha$ is a numerical factor that is on the order of unity. ${ }^{27}$ The quantities $S$ and $g$ are the total spin and the effective Lande $g$ factor of the localized magnetic moments, respectively. The parameter $A_{1}$ is a measure of the contribution of spin scattering to the total magnetoresistance.

The magnetization term in Eq. (2) is given by

$$
M(T, H)=M_{\mathrm{F}}(T, H)+\left[\chi_{p}(T)\right] H,
$$

where $M_{\mathrm{F}}(T, H)$ is the ferromagnetic component due to interacting $\mathrm{Mn}$ ions, $\chi_{p}(T)$ is the high field paramagnetic susceptibility due to noninteracting $\mathrm{Mn}$ ions, $T$ is the temperature, and $H$ is the applied magnetic field.

To explain positive magnetoresistance, a two-band model is utilized. ${ }^{25,28-31}$ In this model, electrical transport occurs 


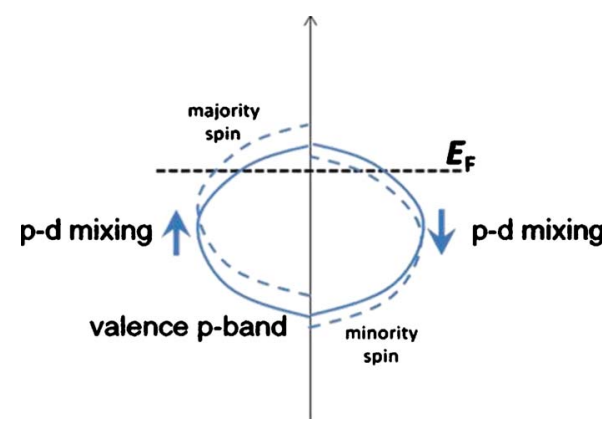

FIG. 1. (Color online) Schematic of the spin-polarized DOS in the case of $p$ - $d$ exchange.

via a valence band that consists of a partially filled hybridized $p-d$ band that is spin split. The resultant two bands have different transport behavior. A schematic for the density of states versus energy is given in Fig. 1. The dashed curves denote the majority and minority carrier bands after kinetic $p$ - $d$ exchange. ${ }^{32,33}$ In the $p$ - $d$ exchange mechanism the lowlying $d$ band hybridizes with the majority $p$ band and pushes it to higher energies above the Fermi level. The higher lying $d$ band hybridizes with the minority $p$ band and thus pushing it away from the Fermi level to lower energies. ${ }^{32}$ This hybridization leads to the spin splitting of the bands. Carriers with different spin polarization have different mobilities. ${ }^{9,10}$

For a two-band model the positive magnetoresistance is given by ${ }^{25,28-30}$

$$
\frac{\Delta \rho}{\rho}=\frac{c^{2} H^{2}}{\left(1+d^{2} H^{2}\right)},
$$

where the fitting parameters $c$ and $d$ are related to the conductivity and mobility of carriers in the two spin-split bands, respectively. Specifically, $c$ and $d$ are related to the band parameters by

$$
\begin{aligned}
& c^{2}=\frac{\sigma_{1} \sigma_{2}\left(\mu_{1}+\mu_{2}\right)^{2}}{\left(\sigma_{1}+\sigma_{2}\right)^{2}}, \\
& d^{2}=\frac{\left(\sigma_{1} \mu_{2}-\sigma_{2} \mu_{1}\right)^{2}}{\left(\sigma_{1}+\sigma_{2}\right)^{2}},
\end{aligned}
$$

where $\mu_{i}$ and $\sigma_{i}$ are the mobilities and conductivities of the carriers in the two bands, respectively. The subscripts 1 and 2 denote the majority-spin and minority-spin carriers, respectively. The relative population of carriers in these two bands is determined by the spin splitting.

\section{EXPERIMENTAL RESULTS AND DISCUSSION}

\section{A. Transverse magnetoresistance}

The dependence of the transverse magnetoresistance on applied magnetic field at temperatures ranging from 1.4 to $298 \mathrm{~K}$ is shown in Fig. 2. The magnetoresistance is given by $\Delta \rho / \rho=\left(\rho-\rho_{0}\right) / \rho_{0}$, where $\rho$ and $\rho_{0}$ are the resistivities in the presence and absence of an external magnetic field. The magnetic field is oriented normal to both the direction of current flow and to the plane of the film. The top panel
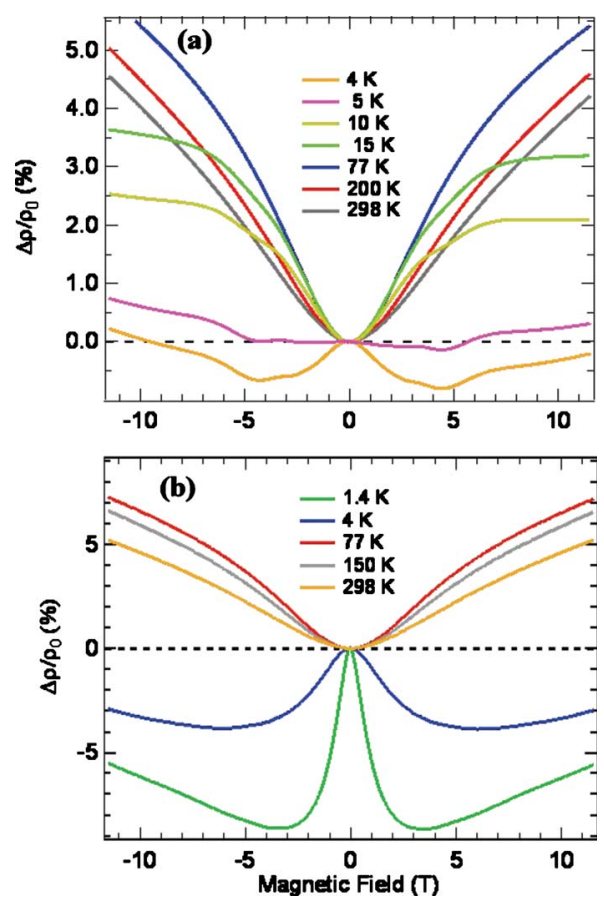

FIG. 2. (Color online) (a) Magnetoresistance data for $\mathrm{In}_{0.98} \mathrm{Mn}_{0.02} \mathrm{Sb}$ and (b) $\mathrm{In}_{0.965} \mathrm{Mn}_{0.035} \mathrm{Sb}$ thin films.

shows the magnetoresistance of the $2 \% \operatorname{In}_{1-x} \mathrm{Mn}_{x} \mathrm{Sb}$ sample while the bottom panel shows it for the $3.5 \%$ sample. As can be seen from Figs. 2(a) and 2(b), the magnetoresistance is negative at low temperatures (below $10 \mathrm{~K}$ ) and decreases in its absolute value as the temperature is elevated. For temperatures above $10 \mathrm{~K}$, the magnetoresistance changes sign and reaches its maximum at $77 \mathrm{~K}$; then the magnitude decreases slowly while remaining positive. The magnetoresistance observed at temperatures below $10 \mathrm{~K}$ has two components: a negative component that dominates up to fields of 3-5 T, then passes through a minimum, and subsequently becomes positive starting with these fields up to $15 \mathrm{~T}$. The lower the Mn concentration or temperature, the lower the field required for the change in slope of magnetoresistance with temperature. In addition, the magnitude of the negative magnetoresistance at low temperatures increases with increasing Mn concentration for a particular magnetic field (e.g., $1 \mathrm{~T}$ ); this indicates that spin scattering decreases with increasing $\mathrm{Mn}$ concentration which is consistent with Eq. (2). We also see from Figs. 2(a) and 2(b) that the magnetoresistance shows a tendency to saturate at higher fields. It is worth noting that the disappearance of the negative magnetoresistance above $10 \mathrm{~K}$ does not represent a magnetic transition from ferromagnetic to paramagnetic states as the $T_{\mathrm{C}}$ for the two films grown under identical conditions was found to be in excess of $400 \mathrm{~K}$ from both SQUID magnetometry and magnetic force microscopy. ${ }^{21}$

To determine the scattering mechanisms responsible for the observed low-temperature magnetoresistance shown in Fig. 2, we performed a least-squares fitting of our data to the expression given by 


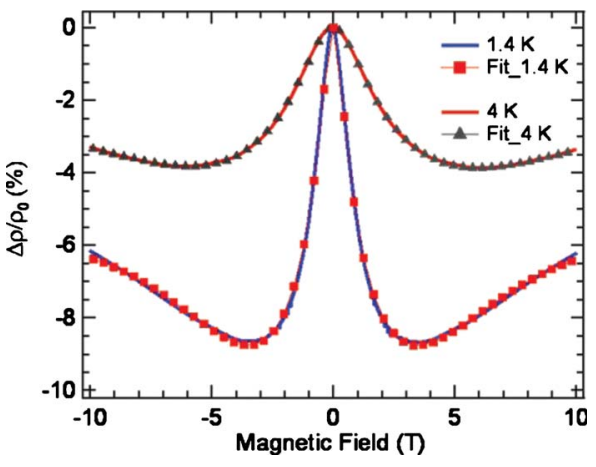

FIG. 3. (Color online) Least-squares fit of the low-temperature magnetoresistance data to Eq. (8) for the $3.5 \%$ sample.

$$
\frac{\Delta \rho}{\rho}=-a^{2} \ln \left(1+b^{2} H^{2}\right)+\frac{c^{2} H^{2}}{\left(1+d^{2} H^{2}\right)}
$$

taking into account both the negative and positive contributions to the magnetoresistance. The four parameters $a, b, c$, and $d$ were treated as fitting parameters. The fitted magnetoresistance agrees very well with the experimental data as seen in Fig. 3. The observed negative magnetoresistance is due to a decrease in spin scattering of holes. Using the values obtained from the fitting procedure it was found that the parameters $a, b, c$, and $d$ have a temperature and concentration dependence (see Table I). Specifically the value of $b$, and hence the absolute magnitude of the negative magnetoresistance, decreases with increasing carrier and dopant concentration while increasing with decreasing temperature (Table II). On the other hand, the parameter $a$ increases with increasing dopant concentration and hence with increasing spin magnetic moment $S$. Cooling the sample from 4 to $1.4 \mathrm{~K}$ increases the absolute magnitude of the negative magnetoresistance which is consistent with Eq. (3). The lowtemperature behavior of our samples is consistent with the magnetic moment model and gives us added confidence with regard to the model described by Eq. (1).

Information about the exchange interaction energy $J$ in InMnSb can be obtained from the magnitude of the parameter $b$ in Eq. (3). ${ }^{34}$ We see from Table II that the value of $b$ decreases from 1.557 to 0.975 for Mn concentrations $x$ $=0.02$ and $x=0.035$, respectively. By substituting the values of $S=5 / 2$ and $g=2$ for the $\mathrm{Mn}^{2+}$ ion into Eq. (3), we find that at a fixed temperature $(4 \mathrm{~K}$, for example), the product $J$ $\times D\left(\varepsilon_{\mathrm{F}}\right)$ (assuming $\left.\alpha=1\right)^{27}$ decreases with increasing $\mathrm{Mn}$ concentration. In addition, Table II shows that the carrier

TABLE I. Values of the fitting parameters to the expression: $\frac{\Delta \rho}{\rho}=-a^{2} \ln \left(1+b^{2} H^{2}\right)+\left[c^{2} H^{2} /\left(1+d^{2} H^{2}\right)\right]$.

\begin{tabular}{ccccccc}
\hline \hline Sample & $x$ & $\begin{array}{c}\text { Temperature } \\
(\mathrm{K})\end{array}$ & $a$ & $b$ & $c$ & $d$ \\
\hline $2 \% \mathrm{Mn}$ & 0.02 & 1.4 & & & & \\
& & 4 & 0.520 & 1.557 & 0.162 & 0.101 \\
$3.5 \% \mathrm{Mn}$ & 0.035 & 1.4 & 1.645 & 2.683 & 0.583 & 0.141 \\
& & 4 & 1.278 & 0.975 & 0.274 & 0.083 \\
\hline \hline
\end{tabular}

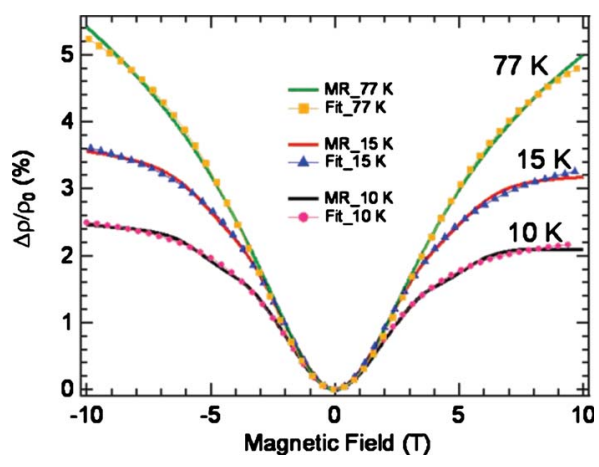

FIG. 4. (Color online) Least-squares fit of the positive magnetoresistance to Eq. (5) for temperatures between 10 and $77 \mathrm{~K}$.

concentration increases with increasing $\mathrm{Mn}$ concentration and implies that the density of states $D\left(\varepsilon_{\mathrm{F}}\right)$ also increases with increasing $\mathrm{Mn}$ concentration. Consequently, it follows that the decrease in the product $J \times D\left(\varepsilon_{\mathrm{F}}\right)$ denotes a reduction in the value of $J$ for increasing Mn concentration. The above observation is explained as follows: by increasing $\mathrm{Mn}$ concentration, the $\mathrm{Mn}$ atoms are closer to one another and hence will lead to an overall reduction in the exchange energy since $J$ depends on the distance of Mn atoms. ${ }^{35}$

The positive magnetoresistance can be explained by employing the two-band conduction model. Results of a leastsquares fit of the magnetoresistance data for the two-band model expression, given by Eq. (5), are shown in Fig. 4 for the $2 \%$ sample. Similar fits were also obtained for the $3.5 \%$ sample (not shown). As can be seen from Eq. (5), the magnitude of the positive magnetoresistance is a function of the parameters $c$ and $d$ that are related to the conductivities and carrier mobilities in the two spin subbands. ${ }^{16}$ The dependence of $\Delta \rho / \rho$ on $H$ increases more slowly than $H^{2}$, increases linearly with $H$, or tends to a saturation value for $d^{2} H^{2} \gg 1$. Thus, the parameter $d$ in Eq. (5) determines, in part, the saturation value of the positive magnetoresistance. The values of the parameter $d$ for the $2 \%$ sample are 0.327 , $0.288,0.202,0.177$, and 0.154 for $10 \mathrm{~K}, 15 \mathrm{~K}, 77 \mathrm{~K}, 150 \mathrm{~K}$, and $298 \mathrm{~K}$, respectively, as summarized in Table III. The dependence of $d$ on temperature is reflected in Fig. 4, where saturation occurs at higher fields for higher temperatures. In addition, we observe from Fig. 4 that the magnitude of the magnetoresistance for a particular field ( $5 \mathrm{~T}$, for example) increases with increasing temperature up to $77 \mathrm{~K}$. Accordingly, the values of $c$ and $d$ will be temperature dependent as shown in Table III. This temperature dependence of the magnetoresistance is attributed to the difference in the mobilities of the spin-spilt bands for the spin-polarized carriers. ${ }^{9,10}$

To relate the values of the fitting parameters $c$ and $d$ to the electrical parameters (Table IV) $\sigma_{i}, \mu_{i}$, and $n_{i}\left(n_{i}\right.$ are the

TABLE II. Concentration dependence of the fitting parameter $b$ at $4 \mathrm{~K}$.

\begin{tabular}{ccc}
\hline \hline $\begin{array}{l}\text { Mn } \\
(\%)\end{array}$ & $\begin{array}{c}\text { Carrier conc. } \\
\left(\mathrm{cm}^{-3}\right)\end{array}$ & $b($ at $4 \mathrm{~K})$ \\
\hline 2.0 & $1.00 \times 10^{19}$ & 1.557 \\
3.5 & $3.00 \times 10^{19}$ & 0.975 \\
\hline
\end{tabular}


TABLE III. Values of the fitting parameters $c$ and $d$ for the expression: $\frac{\Delta \rho}{\rho}=\frac{c^{2} H^{2}}{\left(1+d^{2} H^{2}\right)}$.

\begin{tabular}{ccccc}
\hline \hline \multirow{5}{*}{ Sample } & $x$ & $\begin{array}{c}\text { Temperature } \\
(\mathrm{K})\end{array}$ & $c$ & $d$ \\
\hline \multirow{2}{*}{$2 \% \mathrm{Mn}$} & \multirow{2}{*}{0.02} & 10 & 0.529 & 0.327 \\
& & 15 & 0.550 & 0.288 \\
& & 77 & 0.506 & 0.202 \\
& & 150 & 0.412 & 0.177 \\
& & 298 & 0.357 & 0.154 \\
$3.5 \% \mathrm{Mn}$ & 0.035 & 77 & 0.529 & 0.187 \\
& & 150 & 0.466 & 0.169 \\
& & 298 & 0.376 & 0.149 \\
\hline \hline
\end{tabular}

carrier concentrations), analytic expressions are obtained for $\mu_{i}$ and $\sigma_{i}$ using Eqs. (6) and (7). For a given temperature and Mn concentration these band parameters were obtained from the measured magnetoresistance data using the following derived equations:

$$
\begin{gathered}
\sigma_{i}=\frac{\sigma}{2}\left(1 \pm \sqrt{1-\frac{4 c^{2}}{\left(\mu^{*}\right)^{2}}}\right), \\
\mu_{i}=\frac{\mu^{*} \sigma_{i}}{\sigma} \pm d,
\end{gathered}
$$

where the total conductivity $\sigma_{1}+\sigma_{2}=\sigma$ and the total mobility $\mu_{1}+\mu_{2}=\mu^{*}$. The value of $n_{i}$ is readily obtained from the relation $n_{i}=\sigma_{i} / e \mu_{i}$. The computed values of the total carrier concentration $n=n_{1}+n_{2}$ can then be compared to the experimentally measured values of $n$.

Table $\mathrm{V}$ lists the values of the extracted parameters $n_{1}, \mu_{1}, \sigma_{1}, n_{2}, \mu_{2}, \sigma_{2}$ for the two bands from 10 to $300 \mathrm{~K}$ for an alloy with $x=2 \%$ with applied magnetic field perpendicular to the sample. Clearly, the majority carrier contribution is dominant. With increasing temperature, the values of $\sigma_{1}$ and $\mu_{1}$ increase while $\sigma_{2}$ and $\mu_{2}$ decrease. In addition, there is a very good agreement between the values of $n$ predicted by the model and the measured values from the Hall effect. It is therefore reasonable to conclude from the above analysis that the excellent agreement between the data and the fitting functions support the assumption of a two-band conduction mechanism to describe the positive magnetoresistance [Eq. (5)].

It is important to note the large differences in the magnitudes of carrier concentrations and mobilities for the two
TABLE V. Carrier concentration, conductivity, and mobility in two bands for $\operatorname{In}_{1-x} \mathrm{Mn}_{x} \mathrm{Sb} \quad(x=2 \%)$ and their temperature dependence.

\begin{tabular}{llll}
\hline \hline Two-band parameters & $10 \mathrm{~K}$ & $77 \mathrm{~K}$ & $300 \mathrm{~K}$ \\
\hline Band 1 (majority) & & & \\
$n_{1}\left(\times 10^{19} \mathrm{~cm}^{-3}\right)$ & 1.13 & 1.07 & 0.99 \\
$\sigma_{1}\left(\times 10^{2} 1 / \Omega \mathrm{cm}\right)$ & 2.45 & 2.86 & 3.19 \\
$\mu_{1}\left(\times 10^{2} \mathrm{~cm}^{2} / \mathrm{V} \mathrm{s}\right)$ & 1.35 & 1.67 & 2.01 \\
Band $2\left(\mathrm{minority}^{2}\right.$ & & & \\
$n_{2}\left(\times 10^{16} \mathrm{~cm}^{-3}\right)$ & 7.10 & 8.04 & 4.13 \\
$\sigma_{2}\left(\times 10^{-2} 1 / \Omega \mathrm{cm}\right)$ & 4.03 & 3.11 & 1.07 \\
$\mu_{2}\left(\times 10^{-1} \mathrm{~cm}^{2} / \mathrm{V} \mathrm{s}\right)$ & 3.29 & 2.04 & 1.55 \\
\hline \hline
\end{tabular}

bands listed in Table V. For the majority-spin band 1 the concentration is on the order of $10^{19} \mathrm{~cm}^{-3}$ and for the minority-spin band 2 the concentration is on the order of $10^{17} \mathrm{~cm}^{-3}$. Thus, the magnetoresistance measurements indicate that the alloy shows nearly $100 \%$ spin polarization, where $P=\left(n_{1}-n_{2}\right) / n$. The alloy is nearly a ferromagnetic half metal even at $298 \mathrm{~K} .{ }^{36}$ The carrier mobilities for the two bands also differ by a factor of seven hundred. This suggests that the high-mobility band 1 is mainly $p$-like and the lowmobility band 2 is mainly $d$-like in character.

\section{B. Hall effect and magnetic properties}

The Hall resistivity $\rho_{\mathrm{xy}}$ of the two films was measured over the temperature range of 1.4-298 K. The Hall resistivity of a magnetic semiconductor has an ordinary contribution $\left(\rho^{\mathrm{OH}}\right)$ due to the Lorentz force that is proportional to the external magnetic field $H$, and an anomalous contribution $\left(\rho^{A H}\right)$ often taken to be proportional to the sample magnetization $M$. The total resistivity is given by ${ }^{37}$

$$
\rho_{\mathrm{xy}}=\rho^{O H}+\rho^{A H}=R_{0} H+R_{\mathrm{A}} M,
$$

where $R_{0}$ and $R_{\mathrm{A}}$ are the normal (ordinary) and anomalous Hall coefficients, respectively, $H$ is the applied magnetic field, and $M$ is the component of the magnetization perpendicular to the sample surface. A nonzero value of $R_{\mathrm{A}} M$ at low field indicates that the films are ferromagnetic. ${ }^{5}$

Figure 5(a) shows the Hall resistivity $\left(\rho_{\text {Hall }}\right)$ versus applied magnetic field for the $2 \% \operatorname{In}_{1-x} \mathrm{Mn}_{x} \mathrm{Sb}$ sample measured at different temperatures. The inset shows the Hall resistance $\left(R_{\mathrm{xy}}\right)$ versus field for an InSb sample without Mn doping. We

\begin{tabular}{|c|c|c|c|c|c|c|c|c|c|c|c|}
\hline \multirow[b]{2}{*}{ Mn conc. $(x)$} & \multicolumn{4}{|c|}{$\begin{array}{l}\text { Resistivity } \rho \\
\left(\times 10^{-3} \Omega \mathrm{cm}\right)\end{array}$} & \multicolumn{4}{|c|}{$\begin{array}{l}\text { Carrier concentration } n_{p} \\
\quad\left(\times 10^{19} \mathrm{~cm}^{-3}\right)\end{array}$} & \multicolumn{3}{|c|}{$\begin{array}{l}\text { Mobility } \mu \\
\left(\mathrm{cm}^{2} / \mathrm{V} \mathrm{s}\right)\end{array}$} \\
\hline & $4 \mathrm{~K}$ & $10 \mathrm{~K}$ & $77 \mathrm{~K}$ & $300 \mathrm{~K}$ & $4 \mathrm{~K}$ & $10 \mathrm{~K}$ & $77 \mathrm{~K}$ & $300 \mathrm{~K}$ & $4 \mathrm{~K}$ & $10 \mathrm{~K}$ & $300 \mathrm{~K}$ \\
\hline 0.02 & 4.81 & 4.08 & 3.52 & 3.14 & 1.12 & 1.13 & 1.06 & 0.99 & 116 & 135 & 201 \\
\hline 0.035 & 1.70 & & & 1.05 & 3.26 & & & 3.01 & 113 & & 198 \\
\hline
\end{tabular}
observe from the plot that the Hall resistivity of $\operatorname{In}_{1-x} \mathrm{Mn}_{x} \mathrm{Sb}$

TABLE IV. Electrical parameters of $\mathrm{In}_{1-x} \mathrm{Mn}_{x} \mathrm{Sb}$ at $4 \mathrm{~K}, 10 \mathrm{~K}, 77 \mathrm{~K}$, and $300 \mathrm{~K}$. 

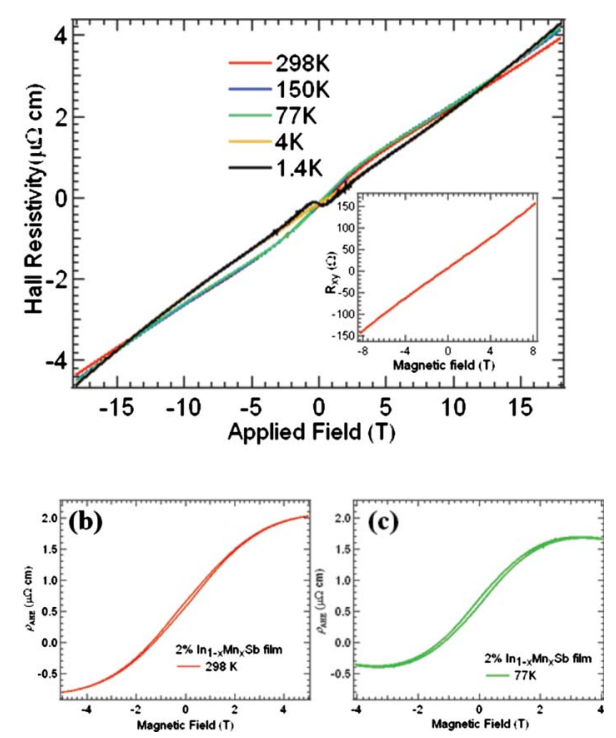

FIG. 5. (Color online) (a) Hall resistivity versus applied magnetic field for the $2 \% \operatorname{In}_{1-x} \mathrm{Mn}_{x} \mathrm{Sb}$ sample measured at 1.4, 4, 77, 150 , and $298 \mathrm{~K}$. Inset shows the Hall resistance vs field for InSb without Mn doping. (b) The anomalous Hall resistivity for the $2 \%$ sample at $77 \mathrm{~K}$ and (c) at $298 \mathrm{~K}$.

at low fields is nonlinear in magnetic field for all measured temperatures. In the low-field regime shown in Figs. 5(b) and 5(c), the AHE dominates the Hall resistivity at all temperatures. The AHE exhibits a hysteresis loop that appears to reflect the magnetization of the sample. Due to the nonlinearity of the Hall resistivity at low magnetic fields, electrical parameters of the $\operatorname{In}_{1-x} \mathrm{Mn}_{x} \mathrm{Sb}$ thin films need to be calculated from the linear portion of the data in the high field range of $10-18 \mathrm{~T}$ where $R_{0} H \gg R_{\mathrm{A}} M$. Electrical parameters were calculated from the linear Hall coefficient in the high field range at 1.4 and $300 \mathrm{~K}$; the parameters are given in Table IV. We observe that the carrier concentration increases with Mn concentration. In addition, the zero-field resistivity decreases with increasing Mn concentration due to increased carrier concentration. Another point of note is that the measured hole concentration of $\sim 10^{19} \mathrm{~cm}^{-3}$ for our films is lower than that reported for $\operatorname{In}_{1-x} \mathrm{Mn}_{x} \mathrm{Sb}$ films grown by LTMBE of $\sim 10^{20} \mathrm{~cm}^{-3} \cdot{ }^{17-19}$ However, the mobility at $300 \mathrm{~K}$ of the MOVPE grown films is higher than that of MBE-grown films.

The nonlinearity of the Hall resistivity presumably results from asymmetric scattering of carriers caused by the spinorbit coupling between mobile charge carriers and localized magnetic moments or Berry phase effects. ${ }^{38,39}$ Since observation of the anomalous Hall effect is often used as a signature to establish ferromagnetism in DMS, it provides evidence for the essential role of hole-mediated coupling between local $\mathrm{Mn}$ moments in long-range ferromagnetic order. ${ }^{5,40}$ A detailed discussion of the origins of the anomalous behavior of the Hall resistivity in $\operatorname{In}_{1-x} \mathrm{Mn}_{x} \mathrm{Sb}$ films grown by MOVPE and its relationship to the observed magnetoresistance will be reported elsewhere. ${ }^{41}$

The magnetization of the two samples was also measured as shown in Fig. 6(a). The saturation magnetization of the $\mathrm{In}_{1-x} \mathrm{Mn}_{x} \mathrm{Sb}$ films is dependent on Mn concentration. For the
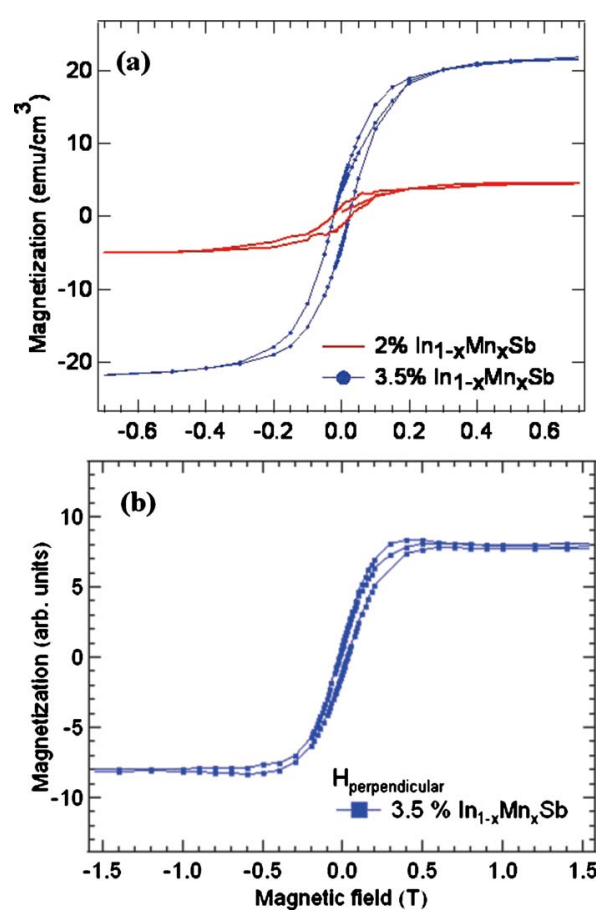

FIG. 6. (Color online) (a) Field dependence of magnetization for the $2 \%$ and $3.5 \%$ samples showing clear hysteresis at $298 \mathrm{~K}$. The magnetic field was applied in the plane of the sample (easy axis). (b) Out-of-plane magnetization vs field of the $3.5 \%$ film.

$2 \%$ film, the measured saturation magnetization $\left(M_{\mathrm{s}}\right)$ is $\sim 4.5 \mathrm{emu} / \mathrm{cm}^{3}$ with a remanence $\left(M_{\mathrm{r}}\right)$ of $\sim 1.6 \mathrm{emu} / \mathrm{cm}^{3}$ and a coercive field $\left(H_{\mathrm{c}}\right)$ of $217 \mathrm{Oe}$. For the $3.5 \%$ film the values of $M_{\mathrm{s}}$ and $M_{\mathrm{r}}$ increase to $\sim 22 \mathrm{emu} / \mathrm{cm}^{3}$ and $\sim 4.6 \mathrm{emu} / \mathrm{cm}^{3}$, respectively. The measured saturation magnetization of $\sim 22 \mathrm{emu} / \mathrm{cm}^{3}$ corresponds to a contribution of $\sim 2.28 \mu_{B}$ per $\mathrm{Mn}$ atoms for the $2 \%$ sample. Figure 6(b) shows the out-of-plane magnetization of the $3.5 \%$ film. The saturation is reached at higher magnetic fields $(\sim 0.5 \mathrm{~T})$ when the magnetization is measured out of plane. Thus, the easy axis of the magnetization in ( In, Mn)Sb thin films is in plane. For comparison, magnetization and Hall measurements carried on InSb without $\mathrm{Mn}$ did not show either ferromagnetism or AHE (see Fig. 7). The InSb sample without $\mathrm{Mn}$ is strongly diamagnetic as is evident from the large negative slope measured at both low and higher magnetic fields. No evidence of hysteresis is observed.

A comparison of the magnetization probed by the anomalous Hall effect and that measured by direct out-of-plane SQUID magnetometry shows nearly the same behavior, as would be expected from Eq. (11). We should note, however, that the saturation field for the anomalous Hall resistivity is much higher than that of SQUID magnetization. This is because SQUID measurements presumably probe the magnetic properties of the entire sample whereas transport measurements probe only regions of higher conductivity, where carrier-mediated magnetic interaction is strongest. ${ }^{42}$

The question of course arises as to the origin of the AHE in the MOVPE films, as to whether or not ferromagnetic precipitates play a role. We have previously reported that XRD and high-resolution TEM indicate that secondary 


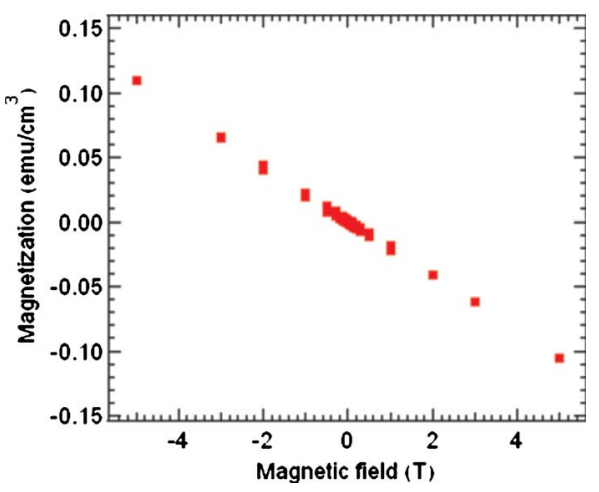

FIG. 7. (Color online) Field dependence of magnetization for an InSb sample without $\mathrm{Mn}$ doping showing very strong diamagnetization.

phases are absent in the films. ${ }^{20}$ In addition, EXAFS analysis indicates that Mn substitutes for In and has tetrahedral coordination as would be expected for a single-phase alloy. Furthermore, ZFC and FC magnetization versus temperature curves show clear reversibility and thus support the homogenous nature of ferromagnetism in the films at room temperature. ${ }^{20,21}$ It has been recently proposed that the ferromagnetism in InMnSb is stabilized by a Ruderman-KittelKasuya-Yosida mechanism and with correlated substitution of magnetic impurities. ${ }^{43,44}$ In this case, substitutional Mn acceptor complexes are the source of both free holes and the localized spins that lead to a high $T_{\mathrm{C}}$. Thus, the observation of hysteresis loops in both the Hall and SQUID measurements that persist above room temperature indicates that the ferromagnetism is attributable to the single-phase alloy and not due to isolated ferromagnetic Mn precipitates in a nonmagnetic semiconductor host.

\section{SUMMARY AND CONCLUSIONS}

In summary, we have described the magnetotransport properties of InMnSb epitaxial thin films on semi-insulating GaAs grown using MOVPE. At temperatures below $10 \mathrm{~K}$, a negative magnetoresistance dominates the magnetotransport; the magnitude of magnetoresistance depends on the Mn concentration. We attribute the negative magnetoresistance to a decrease in spin-dependent scattering of holes by localized $\mathrm{Mn}$ magnetic moments. At temperatures above $10 \mathrm{~K}$, the negative magnetoresistance is negligible and a positive magnetoresistance dominates the magnetotransport. We have analyzed the positive magnetoresistance in terms of a twoband model in which the bands consist of spin-spilt hybridized $p-d$ subbands. Calculations based on the magnetoresistance and its field dependence indicate that the carriers are highly spin polarized. Hall effect measurements indicate an anomalous resistivity component at room temperature, indicating existence of ferromagnetic order in the alloy. Furthermore, magnetization measurements reveal distinct hysteresis loops at room temperature and confirm that the single-phase films are ferromagnetic.

\section{ACKNOWLEDGMENTS}

This work is supported by the National Science Foundation (NSF) under Grant No. DMR-0804479, the Air Force Office of Scientific Research (AFOSR) under Grant No. FA9550-07, and the National High Magnetic Field Laboratory (NHMFL) through NSF cooperative Agreement No. DMR-0654118. This work also made use of the Central Facilities supported by the MRSEC program of the NSF (Grant No. DMR-0520513) at the Materials Research Center of Northwestern University.
*Corresponding author; b-wessels@ northwestern.edu

${ }^{1}$ D. D. Awschalom and M. E. Flatté, Nat. Phys. 3, 153 (2007).

2 J. G. Braden, J. S. Parker, P. Xiong, S. H. Chun, and N. Samarth, Phys. Rev. Lett. 91, 056602 (2003).

${ }^{3}$ R. P. Panguluri, B. Nadgorny, T. Wojtowicz, X. Liu, and J. K. Furdyna, Appl. Phys. Lett. 91, 252502 (2007).

${ }^{4}$ H. Ohno, Science 291, 840 (2001).

${ }^{5}$ Modern Aspects of Spin Physics, Lecture Notes in Physics Vol. 712, edited by W. Pötz, J. Fabian, and U. Hohenester (Springer, Berlin, 2006).

${ }^{6}$ C. Haas, Phys. Rev. 168, 531 (1968).

${ }^{7}$ P. G. de Gennes and J. Friedel, J. Phys. Chem. Solids 4, 71 (1958).

${ }^{8}$ C. Michel, P. J. Klar, S. D. Baranovskii, and P. Thomas, Phys. Rev. B 69, 165211 (2004).

${ }^{9}$ M. Foygel and A. G. Petukhov, Phys. Rev. B 76, 205202 (2007).

${ }^{10}$ M. Foygel, J. Niggemann, and A. G. Petukhov, IEEE Trans. Magn. 43, 3040 (2007).

${ }^{11}$ H. Ohno, H. Munekata, S. von Molnár, and L. L. Chang, J. Appl. Phys. 69, 6103 (1991).

${ }^{12}$ H. Munekata, Mater. Sci. Eng., B 31, 151 (1995).
${ }^{13}$ Y. Iye, A. Oiwa, A. Endo, S. Katsumoto, F. Matsukura, A. Shen, H. Ohno, and H. Munekata, Mater. Sci. Eng., B 63, 88 (1999).

${ }^{14}$ H. Ohno, H. Munekata, T. Penney, S. von Molnár, and L. L. Chang, Phys. Rev. Lett. 68, 2664 (1992).

${ }^{15}$ S. J. May, A. J. Blattner, and B. W. Wessels, Phys. Rev. B 70, 073303 (2004).

${ }^{16}$ J. A. Peters and B. W. Wessels, Physica E 42, 1447 (2010).

${ }^{17}$ T. Wojtowicz, W. L. Lim, X. Liu, G. Cywinski, M. Kutrowski, L. V. Titova, K. Yee, M. Dobrowolska, J. K. Furdyna, K. M. Yu, W. Walukiewicz, G. B. Kim, M. Cheon, X. Chen, S. M. Wang, H. Luo, I. Vurgaftman, and J. R. Meyer, Physica E 20, 325 (2004).

${ }^{18} \mathrm{~S}$. Yanagi, K. Kuga, T. Slupinski, and H. Munekata, Physica E 20, 333 (2004).

${ }^{19}$ M. Csontos, T. Wojtowicz, X. Liu, M. Dobrowolska, B. Janko, J. K. Furdyna, and G. Mihaly, Phys. Rev. Lett. 95, 227203 (2005).

${ }^{20}$ N. D. Parashar, N. Rangaraju, V. K. Lazarov, S. Xie, and B. W. Wessels, Phys. Rev. B 81, 115321 (2010).

${ }^{21}$ N. D. Parashar, D. J. Keavney, and B. W. Wessels, Appl. Phys. Lett. 95, 201905 (2009).

${ }^{22}$ N. D. Parashar, Q. Ma, and B. W. Wessels (unpublished).

${ }^{23}$ Y. Toyozawa, J. Phys. Soc. Jpn. 17, 986 (1962). 
${ }^{24}$ K. Yosida, Phys. Rev. 107, 396 (1957).

${ }^{25}$ R. P. Khosla and J. R. Fischer, Phys. Rev. B 2, 4084 (1970).

${ }^{26}$ J. Kondo, Prog. Theor. Phys. 32, 37 (1964); 34, 204 (1965).

${ }^{27}$ J. A. Appelbaum, Phys. Rev. 154, 633 (1967).

${ }^{28}$ E. H. Sondheimer and A. H. Wilson, Proc. R. Soc. London, Ser. A 190, 435 (1947).

${ }^{29}$ S. M. Watts, S. Wirth, S. von Molnar, A. Barry, and J. M. D. Coey, Phys. Rev. B 61, 9621 (2000).

${ }^{30}$ J. L. Olsen, Electron Transport in Metals (Interscience, New York, 1962).

${ }^{31}$ A. B. Pippard, Magnetoresistance in Metals (Cambridge University Press, Cambridge, 1988).

${ }^{32}$ C. Zener, Phys. Rev. 81, 440 (1951).

${ }^{33}$ B. Belhadji, L. Bergqvist, R. Zeller, P. H. Dederichs, K. Sato, and H. Katayama-Yoshida, J. Phys.: Condens. Matter 19, 436227 (2007).

${ }^{34}$ D. G. Andrianov, G. V. Lazareva, A. S. Savel'ev, V. I. Selyanina, and V. I. Fistul, Sov. Phys. Semicond. 9, 1026 (1976).

${ }^{35}$ R. W. Lynch, J. Chem. Phys. 47, 5180 (1967).
${ }^{36}$ J. M. D. Coey and S. Sanvito, J. Phys. D: Appl. Phys. 37, 988 (2004).

${ }^{37}$ L. Chien and C. Westgate, The Hall Effect and Its Applications (Plenum, New York, 1980).

${ }^{38}$ T. Dietl, F. Matsukura, H. Ohno, J. Cibert, and D. Ferrand, Proc. NATO Workshop Recent Trends in Theory of Physical Phenomena in High Magnetic Fields, edited by I. Vagner et al. (Kluwer, Dordrecht, 2003), p. 197.

${ }^{39}$ T. Jungwirth, Qian Niu, and A. H. MacDonald, Phys. Rev. Lett. 88, 207208 (2002).

${ }^{40}$ H. Ohno, Science 281, 951 (1998).

${ }^{41}$ N. D. Parashar, N. Rangaraju, J. A. Peters, and B. W. Wessels (unpublished).

${ }^{42}$ F. Matsukura and H. Ohno, in Nanomagnetism and Spintronics, edited by Teruya Shinjo (Elsevier Science, 2009).

${ }^{43}$ B. W. Wessels, New J. Phys. 10, 055008 (2008).

${ }^{44}$ E. Z. Meilikhov, Phys. Rev. B 75, 045204 (2007). 